\title{
ZEROS OF THE WRONSKIAN AND RENORMALIZED OSCILLATION THEORY
}

\author{
By F. Gesztesy, B. Simon, and G. Teschl
}

\begin{abstract}
For general Sturm-Liouville operators with separated boundary conditions, we prove the following: If $E_{1,2} \in \mathbb{R}$ and if $u_{1,2}$ solve the differential equation $H u_{j}=E_{j} u_{j}, j=1,2$ and respectively satisfy the boundary condition on the left/right, then the dimension of the spectral projection $P_{\left(E_{1}, E_{2}\right)}(H)$ of $H$ equals the number of zeros of the Wronskian of $u_{1}$ and $u_{2}$.
\end{abstract}

1. Introduction. For over one hundred and fifty years, oscillation theorems for second-order differential equations have fascinated mathematicians. Originating with Sturm's celebrated memoir [20], and extended in a variety of ways by Bôcher [2] and others, a large body of material has been accumulated since then (thorough treatments can be found, e.g., in [4],[13],[18],[19], and the references therein). In this paper we'll add a new wrinkle to oscillation theory by showing that zeros of Wronskians can be used to count eigenvalues in situations where a naive use of oscillation theory would give $\infty-\infty$.

To set the stage, we'll consider operators on $L^{2}((a, b) ; r d x)$ with $a<b$ in $[-\infty, \infty]$ of the form

$$
(\tau u)(x)=r(x)^{-1}\left[-\left(p(x) u^{\prime}(x)\right)^{\prime}+q(x) u(x)\right],
$$

where

$$
r, p^{-1}, q \in L_{\mathrm{loc}}^{1}((a, b)) \text { are real-valued and } r, p>0 \text { a.e. on }(a, b) \text {. }
$$

We'll use $\tau$ to describe the formal differential expression and $H$ the operator given by $\tau$ with separated boundary conditions at $a$ and/or $b$.

If $a$ (resp. $b$ ) is finite and $q, p^{-1}, r$ are in addition integrable near $a$ (resp. $b$ ), we'll say $a$ (resp. $b$ ) is a regular end point. We'll say $\tau$ respectively $H$ is regular if both $a$ and $b$ are regular. As is usual, ([6], section XIII.2; [15], section 17; [22], chapter 3), we consider the local domain

$$
D_{\mathrm{loc}}=\left\{u \in A C_{\mathrm{loc}}((a, b)) \mid p u^{\prime} \in A C_{\mathrm{loc}}((a, b)), \tau u \in L_{\mathrm{loc}}^{2}((a, b) ; r d x)\right\},
$$

Manuscript received May 3, 1995; revised August 16, 1995.

Research supported in part by NSF Grant DMS-9401491.

American Journal of Mathematics 118 (1996), 571-594. 
where $A C_{\mathrm{loc}}((a, b))$ is the set of integrals of $L_{\mathrm{loc}}^{1}$ functions (i.e., the set of locally absolutely continuous functions) on $(a, b)$. General ODE theory shows that for any $E \in \mathbb{C}, x_{0} \in(a, b)$, and $(\alpha, \beta) \in \mathbb{C}^{2}$, there is a unique $u \in D_{\text {loc }}$ such that $-\left(p u^{\prime}\right)^{\prime}+q u-E r u=0$ a.e. on $x \in(a, b)$ and $\left(u\left(x_{0}\right),\left(p u^{\prime}\right)\left(x_{0}\right)\right)=(\alpha, \beta)$.

The maximal and minimal operators are defined by taking

$$
D\left(T_{\max }\right)=\left\{u \in L^{2}((a, b) ; r d x) \cap D_{\mathrm{loc}} \mid \tau u \in L^{2}((a, b) ; r d x)\right\},
$$

with

$$
T_{\max } u=\tau u .
$$

$T_{\min }$ is the operator closure of $T_{\max }\left\lceil D_{\text {loc }} \cap\{u\right.$ has compact support in $(a, b)\}$. Then $T_{\min }$ is symmetric and $T_{\min }^{*}=T_{\max }$.

According to the Weyl theory of self-adjoint extensions ([6], section XIII.6; [15], section 18; [16], appendix to X.1; [21], section 8.4; [22], chapters 4 and 5), the deficiency indices of $T_{\min }$ are $(0,0)$ or $(1,1)$ or $(2,2)$ depending on whether it is limit point at both, one or neither end point. Moreover, the self-adjoint extensions can be described in terms of Wronskians ([6], section XIII.2; [15], sections 17 and 18; [21], section 8.4; [22], chapter 3). Define

$$
W\left(u_{1}, u_{2}\right)(x)=u_{1}(x)\left(p u_{2}^{\prime}\right)(x)-\left(p u_{1}^{\prime}\right)(x) u_{2}(x) .
$$

Then if $T_{\min }$ is limit point at both ends, $T_{\min }=T_{\max }=H$. If $T_{\min }$ is limit point at $b$ but not at $a$, for $H$ any self-adjoint extension of $T_{\min }$, if $\varphi_{-}$is any function in $D(H) \backslash D\left(T_{\min }\right)$, then

$$
D(H)=\left\{u \in D\left(T_{\max }\right) \mid W\left(u, \varphi_{-}\right)(x) \rightarrow 0 \text { as } x \downarrow a\right\} .
$$

Finally, if $u_{1}$ is limit circle at both ends, the operators $H$ with separated boundary conditions are those for which we can find $\varphi_{ \pm} \in D(H), \varphi_{+} \equiv 0$ near $a, \varphi_{-} \equiv 0$ near $b$, and $\varphi_{ \pm} \in D(H) \backslash D\left(T_{\min }\right)$. In that case,

$$
D(H)=\left\{u \in D\left(T_{\max }\right) \mid W\left(u, \varphi_{-}\right)(x) \rightarrow 0 \text { as } x \downarrow a, W\left(u, \varphi_{+}\right)(x) \rightarrow 0 \text { as } x \uparrow b\right\} .
$$

Of course, if $H$ is regular, we can just specify the boundary conditions by taking values at $a, b$ since by regularity any $u \in D\left(T_{\max }\right)$ has $u, p u^{\prime}$ continuous on $[a, b]$ (cf. (A.4)). It follows from this analysis that

Proposition 1.1. If $u_{1,2} \in D(H)$, then $W\left(u_{1}, u_{2}\right)(x) \rightarrow 0$ as $x \rightarrow$ a or $b$.

We'll call such operators SL operators (for Sturm-Liouville, but SL includes separated boundary conditions (if necessary)).

It will be convenient to write $\ell_{-}=a, \ell_{+}=b$. 
Throughout this paper we will denote by $\psi_{ \pm}(z, x) \in D_{\text {loc }}$ solutions of $\tau \psi=z \psi$ so that $\psi_{ \pm}(z, \cdot)$ is $L^{2}$ at $\ell_{ \pm}$and $\psi_{ \pm}(z, \cdot)$ satisfies the appropriate boundary condition at $\ell_{ \pm}$in the sense that for any $u \in D(H), \lim _{x \rightarrow \ell_{ \pm}} W\left(\psi_{ \pm}(z), u\right)(x)=0$. If $\psi_{ \pm}(z, \cdot)$ exist, they are unique up to constant multiples. In particular, $\psi_{ \pm}(z, \cdot)$ exist for $z$ not in the essential spectrum of $H$ and we can assume them to be holomorphic with respect to $z$ in $\mathbb{C} \backslash \operatorname{spec}(H)$ and real for $z \in \mathbb{R}$. One can choose

$$
\psi_{ \pm}(z, x)=\left((H-z)^{-1} \chi_{(c, d)}\right)(x) \quad \text { for } x_{<c}^{>d}, \quad a<c<d<b
$$

and uniquely continue $\psi_{ \pm}(z, x)$ for $x_{<c}^{>d}$. Here $(H-z)^{-1}$ denotes the resolvent of $H$ and $\chi_{\Omega}$ the characteristic function of the set $\Omega \subseteq \mathbb{R}$. Clearly, we can include a finite number of isolated eigenvalues in the domain of holomorphy of $\psi_{ \pm}$by removing the corresponding poles. Moreover, to simplify notations, all solutions $u$ of $\tau u=E u$ are understood to be not identically vanishing and solutions associated with real values of the spectral parameter $E$ are assumed to be real-valued in this paper. Thus if $E$ is real and in the resolvent set for $H$ or an isolated eigenvalue, we are guaranteed solutions that obey the boundary conditions at $a$ or $b$. It can happen if $E$ is in the essential spectrum that such solutions do not exist, or it may happen that they do. In Theorems 1.3, 1.4 below, we'll explicitly assume such solutions exist for the energies of interest; if these energies are not in the essential spectrum, that is automatically fulfilled.

With these preliminaries out of the way, we can describe a theorem Hartman proves in [10] which gives an eigenvalue count in some cases where oscillation theory would naively give $\infty-\infty$ (see Weidmann [22], chapter 14 for some results when $\tau$ is limit circle at $b$ ). In fact, we have slightly generalized the theorem in order to include, for instance, certain singular cases like radial Schrödinger operators on $(0, \infty)$ with potentials singular near 0 (we shall give a proof in Section 7).

THEOREM 1.2. Let $H$ be an SL operator on $(a, b)$ which is non-oscillatory at $E_{2}$ near $a$ and limit point $a t b$ and suppose $E_{1}<E_{2}$. Let $u_{1}$ (resp. $\left.u_{2}\right)$ be $\psi_{-}\left(E_{1}\right)$ (resp. $\left.\psi_{-}\left(E_{2}\right)\right)$. Let $N(c), c \in(a, b)$ denote the number of zeros of $u_{1}$ in $(a, c)$ minus the number of zeros of $u_{2}$ in $(a, c)$. Let $P_{\Omega}(H)$ be the spectral projection of $H$ corresponding to the Borel set $\Omega \subseteq \mathbb{R}$. Then, if $\tau$ is oscillatory at $E_{2}$ near $b$,

$$
\operatorname{dim} \operatorname{Ran} P_{\left(E_{1}, E_{2}\right)}(H)=\frac{\lim }{c \uparrow b} N(c),
$$

and if $\tau$ is non-oscillatory at $E_{2}$ near $b$,

$$
\operatorname{dim} \operatorname{Ran} P_{\left[E_{1}, E_{2}\right)}(H)=\lim _{c \uparrow b} N(c) .
$$

Theorem 1.2 is a bit more general than Hartman's result in [10] (see also [9],[11]) since we assume $H$ to be non-oscillatory at $E_{2}$ near $a$ while Hartman 
assumes $H$ to be regular at $a$. If $\tau$ is oscillatory at $E_{2}$ near $b$ (i.e., $u_{2}$ has infinitely many zeros near $b), N(c)$ is not constant for large $c$ but instead varies between $N_{0}$ and $N_{0}+1$. This result leaves several questions open: What happens if $H$ is limit circle at $b$ or in the case where $H$ is not regular at either end (e.g., the important case of the real line $(a, b)=(-\infty, \infty))$ ? Moreover, it isn't clear when $c$ is so large that $\frac{\lim }{c \uparrow b} N(c)$ has been reached. It would be better if we could actually count something analogous to the zero count in ordinary oscillation theory. Our goal in this paper is to prove such theorems.

The key is to look at zeros of the Wronskian. That zeros of the Wronskian are related to oscillation theory is indicated by an old paper of Leighton [14], who noted that if $u_{j}, p u_{j}^{\prime} \in A C_{\mathrm{loc}}((a, b)), j=1,2$ and $u_{1}$ and $u_{2}$ have a nonvanishing Wronskian $W\left(u_{1}, u_{2}\right)$ in $(a, b)$, then their zeros must intertwine each other. (In fact, $p u_{1}^{\prime}$ must have opposite signs at consecutive zeros of $u_{1}$, so by nonvanishing of $W, u_{2}$ must have opposite signs at consecutive zeros of $u_{1}$ as well. Interchanging the role of $u_{1}$ and $u_{2}$ yields strict interlacing of their zeros.) Moreover, let $E_{1}<E_{2}$ and $\tau u_{j}=E_{j} u_{j}, j=1,2$. If $x_{0}, x_{1}$ are two consecutive zeros of $u_{1}$, then the number of zeros of $u_{2}$ inside $\left(x_{0}, x_{1}\right)$ is equal to the number of zeros of the Wronskian $W\left(u_{1}, u_{2}\right)$ plus one (cf. Theorem 7.4). Hence the Wronskian comes with a builtin renormalization counting the additional zeros of $u_{2}$ in comparison to $u_{1}$. In particular, this avoids taking limits of the type (1.5a).

We'll let $W_{0}\left(u_{1}, u_{2}\right)$ be the number of zeros of the Wronskian in the open interval $(a, b)$ not counting multiplicities of zeros. Given $E_{1}<E_{2}$, we let $N_{0}\left(E_{1}, E_{2}\right)=$ $\operatorname{dim} \operatorname{Ran} P_{\left(E_{1}, E_{2}\right)}(H)$ be the dimension of the spectral projection $P_{\left(E_{1}, E_{2}\right)}(H)$ of $H$. Our main results are the following two theorems:

THEOREM 1.3. Suppose $E_{1}<E_{2}$. Let $u_{1}=\psi_{-}\left(E_{1}\right)$ and $u_{2}=\psi_{+}\left(E_{2}\right)$. Then

$$
W_{0}\left(u_{1}, u_{2}\right)=N_{0}\left(E_{1}, E_{2}\right)
$$

THEOREM 1.4. Suppose $E_{1}<E_{2}$. Let $u_{1}=\psi_{-}\left(E_{1}\right)$ and $u_{2}=\psi_{-}\left(E_{2}\right)$. Then either

$$
W_{0}\left(u_{1}, u_{2}\right)=N_{0}\left(E_{1}, E_{2}\right)
$$

or

$$
W_{0}\left(u_{1}, u_{2}\right)=N_{0}\left(E_{1}, E_{2}\right)-1 \text {. }
$$

If either $N_{0}=0$ or $H$ is limit point at $b$, then (1.6) holds.

We'll see that if $b$ is a regular point and $E_{2}>e>E_{1}$ with $e$ an eigenvalue and $\left|E_{2}-E_{1}\right|$ is small, then (1.7) holds rather than (1.6). We'll also see that if $u_{1,2}$ are arbitrary solutions of $\tau u_{j}=E_{j} u_{j}, j=1,2$, then, in general, $\left|W_{0}-N_{0}\right| \leq 2$ (this means that if one of the quantities is infinite, the other is as well) and any of 
$0, \pm 1, \pm 2$ can occur for $W_{0}-N_{0}$. Especially, if either $E_{1}$ or $E_{2}$ is in the interior of the essential spectrum of $H$ (or $\operatorname{dim} \operatorname{Ran} P_{\left(E_{1}, E_{2}\right)}(H)=\infty$ ), then $W_{0}\left(u_{1}, u_{2}\right)=\infty$ for any $u_{1}$ and $u_{2}$ satisfying $\tau u_{j}=E_{j} u_{j}, j=1,2$ (cf. Theorem 7.3).

Zeros of the Wronskians have two properties that are critical to these results: First, zeros are precisely points where the Prüfer angles for $u_{1}$ and $u_{2}$ are equal $(\bmod \pi)$. Second, if $\psi_{-} \in D_{\mathrm{loc}}$ and $\psi_{+} \in D_{\mathrm{loc}}$ satisfy the boundary conditions at $a, b$, respectively, and $W\left(\psi_{-}, \psi_{+}\right)\left(x_{0}\right)=0$ and if $\left(\psi_{+}\left(x_{0}\right),\left(p \psi_{+}^{\prime}\right)\left(x_{0}\right)\right) \neq(0,0)$, then there is a $\gamma$ such that

$$
\eta(x)= \begin{cases}\psi_{-}(x), & x \leq x_{0} \\ \gamma \psi_{+}(x), & x \geq x_{0}\end{cases}
$$

satisfies $\eta \in D(H)$ and

$$
H \eta(x)= \begin{cases}\left(\tau \psi_{-}\right)(x), & x \leq x_{0} \\ \gamma\left(\tau \psi_{+}\right)(x), & x \geq x_{0}\end{cases}
$$

We'll explore these properties further in Propositions 3.1 and 3.2.

Section 2 provides a short proof of the ordinary oscillation theorem in the regular case following the method in Courant-Hilbert ([5], page 454). Even though this result is well-known (see, e.g., [1], theorem 8.4.5 and [22], theorem 14.10 which describes the singular case as well), we include it here since our overall strategy in this paper is patterned after this proof: A variational argument will show $N_{0} \geq W_{0}$ in Section 6, and a comparison-type argument in Sections 4 and 5 will prove $N_{0} \leq W_{0}$. Explicitly, in Section 5 we'll show

THEOREM 1.5. Let $E_{1}<E_{2}$. If $u_{1}=\psi_{-}\left(E_{1}\right)$ and either $u_{2}=\psi_{+}\left(E_{2}\right)$ or $\tau u_{2}=$ $E_{2} u_{2}$ and $H$ is limit point at $b$, then

$$
W_{0}\left(u_{1}, u_{2}\right) \geq \operatorname{dim} \operatorname{Ran} P_{\left(E_{1}, E_{2}\right)}(H) .
$$

In Section 6, we'll prove that

THEOREM 1.6. Let $E_{1}<E_{2}$. Let either $u_{1}=\psi_{+}\left(E_{1}\right)$ or $u_{1}=\psi_{-}\left(E_{1}\right)$ and either $u_{2}=\psi_{+}\left(E_{2}\right)$ or $u_{2}=\psi_{-}\left(E_{2}\right)$. Then

$$
W_{0}\left(u_{1}, u_{2}\right) \leq \operatorname{dim} \operatorname{Ran} P_{\left(E_{1}, E_{2}\right)}(H) .
$$

Remark. Of course, by reflecting about a point $c \in(a, b)$, Theorems 1.3-1.5 hold for $u_{1}=\psi_{+}\left(E_{1}\right)$ and $u_{2}=\psi_{-}\left(E_{2}\right)$ (and either $N_{0}=0$ or $H$ is limit point at $a$ in the corresponding analog of Theorem 1.4 yields (1.6) and similarly, $\tau u_{2}=E_{2} u_{2}$ and $H$ is limit point at $a$ yields the conclusion in the corresponding analog of Theorem 1.5).

In Section 7, we provide a number of comments, examples, and extensions including: 
THEOREM 1.7. Let $E_{1,2} \in \mathbb{R}, E_{1} \neq E_{2}, \tau u_{j}=E_{j} u_{j}, j=1,2, \tau \mathrm{V}_{2}=E_{2} \mathrm{~V}_{2}$. Then $\left|W_{0}\left(u_{1}, u_{2}\right)-W_{0}\left(u_{1}, \mathrm{v}_{2}\right)\right| \leq 1$.

In addition, Theorem 7.5 addresses the problem of finite versus infinite total number of eigenvalues in essential spectral gaps of $H$.

It is easy to see that Theorems 1.5, 1.6, and 1.7 imply Theorems 1.3 and 1.4.

Some facts on quadratic forms are collected in the appendix.

Our interest in this subject originated in attempts to provide a general construction of isospectral potentials for one-dimensional Schrödinger operators (see [8]) following previous work by Finkel, Isaacson, and Trubowitz [7] (see also [3]) in the case of periodic potentials. In fact, in the special case of periodic Schrödinger operators $H_{p}$, the nonvanishing of $W\left(u_{1}, u_{2}\right)(x)$ for Floquet solutions $u_{1}=\psi_{\varepsilon_{1}}\left(E_{1}\right), u_{2}=\psi_{\varepsilon_{2}}\left(E_{2}\right), \varepsilon_{1,2} \in\{+,-\}$ of $H_{p}$, for $E_{1}$ and $E_{2}$ in the same spectral gap of $H_{p}$, is proven in [7].

2. Oscillation theory. For background, we recall the following:

THEOREM 2.1. ([22], theorem 4.10) Let $H$ be an SL operator which is bounded from below. If $e_{1}<\cdots<e_{n}<\cdots$ are its eigenvalues below the essential spectrum and $\psi_{1}, \ldots, \psi_{n}, \ldots$ its eigenfunctions, then $\psi_{n}$ has $n-1$ zeros in $(a, b)$. All eigenvalues of $H$ are simple.

Remarks. (i) Those used to thinking of the Dirichlet boundary condition case need to be warned that it is not in general true that if $E$ is not an eigenvalue of $H$, then the number of zeros, $Z$, of $\psi_{ \pm}(E)$ is the number, $N(E)$, of eigenvalues less than $E$. In general, all one can say is $N=Z$ or $N=Z+1$.

(ii) In the special case where $\tau$ is regular at $a$ and $b$, any associated SL operator $H$ is well-known to be bounded from below with compact resolvent (see, e.g., [1], theorem 8.4.5; [22], theorem 13.2). Thus Theorem 2.1 applies to the regular case (to be used in our proof of Proposition 4.1).

The first part of the proposition below is a simple integration by parts and the second follows from the first.

Proposition 2.2. Let $E_{1} \leq E_{2}$ and $\tau u_{j}=E_{j} u_{j}, j=1,2$. Then for $a<c<d<b$,

$$
W\left(u_{1}, u_{2}\right)(d)-W\left(u_{1}, u_{2}\right)(c)=\left(E_{2}-E_{1}\right) \int_{c}^{d} u_{1}(x) u_{2}(x) r(x) d x
$$

In particular, $W\left(u_{1}, u_{2}\right) \in A C_{\mathrm{loc}}((a, b))$ and

$$
\frac{d W\left(u_{1}, u_{2}\right)}{d x}(x)=\left(E_{2}-E_{1}\right) r(x) u_{1}(x) u_{2}(x) \quad \text { a.e. }
$$

If the problem is regular at a (resp. $b$ ), we can take $c$ resp. d) equal to $a$ (resp. $b$ ). In the general case we can take the limit $c \downarrow a$ (resp. $d \uparrow b$ ) in (2.1) if $u_{1}$ and $u_{2}$ are $L^{2}$ near $a$ resp. $b$ ). 
COROLlaRY 2.3. Let $E_{1}<E_{2}$ and $\tau u_{j}=E_{j} u_{j}, j=1,2$. Suppose at each end of $[c, d], a<c<d<b$ either $W\left(u_{1}, u_{2}\right)=0$ or $u_{1}=0$. If $\lim _{x \downarrow a} W\left(u_{1}, u_{2}\right)(x)=0$ (resp. $\left.\lim _{x \uparrow b} W\left(u_{1}, u_{2}\right)(x)=0\right)$, we also consider $c=a($ resp. $d=b)$. Then $u_{2}$ must vanish at least once in $(c, d)$.

Proof. By decreasing $d$ to the first zero of $u_{1}$ in $(c, d]$ (and perhaps flipping signs), we can suppose $u_{1}>0$ on $(c, d)$. If $u_{2}$ has no zeros in $(c, d)$, we can suppose $u_{2}>0$ on $(c, d)$ again by perhaps flipping signs. At each end point, $W\left(u_{1}, u_{2}\right)$ vanishes or else $u_{1}=0, u_{2}>0$, and $u_{1}^{\prime}(c)>0$ (or $u_{1}^{\prime}(d)<0$ ). Thus, $W\left(u_{1}, u_{2}\right)(c) \geq 0, W\left(u_{1}, u_{2}\right)(d) \leq 0$. Since the right side of $(2.1)$ is positive, this is inconsistent with (2.1).

Proof of Theorem 2.1. We first prove that $\psi_{n}$ has at least $n-1$ zeros and then that if $\psi_{n}$ has $m$ zeros, then $\left(-\infty, e_{n}\right.$ ] has at least $(m+1)$ eigenvalues. If $\psi_{n}$ has $m$ zeros at $x_{1}, x_{2}, \ldots, x_{m}$ and we let $x_{0}=a, x_{m+1}=b$, then by Corollary 2.3, $\psi_{n+1}$ must have at least one zero in each of $\left(x_{0}, x_{1}\right),\left(x_{1}, x_{2}\right), \ldots,\left(x_{m}, x_{m+1}\right)$, that is, $\psi_{n+1}$ has at least $m+1$ zeros. It follows by induction that $\psi_{n}$ has at least $n-1$ zeros.

On the other hand, if an eigenfunction $\psi_{n}$ has $m$ zeros, define for $j=0, \ldots, m$, $x_{0}=a, x_{m+1}=b$,

$$
\eta_{j}(x)=\left\{\begin{array}{ll}
\psi_{n}(x), & x_{j} \leq x \leq x_{j+1} \\
0, & \text { otherwise }
\end{array} \quad, \quad 0 \leq j \leq m .\right.
$$

Then $\eta_{j}$ is absolutely continuous with $p \eta_{j}^{\prime}$ piecewise continuous so $\eta_{j}$ is in the form domain of $H$ (see (A.6)) and $\left\langle|H|^{1 / 2} \eta_{j}, \operatorname{sgn}(H)|H|^{1 / 2} \eta_{j}\right\rangle=e_{n}\left\|\eta_{j}\right\|$ (where $\langle\cdot, \cdot\rangle$ and $\|\cdot\|$ denote the scalar product and norm in $L^{2}((a, b) ; r d x)$. Thus if $\eta=\sum_{j=0}^{m} c_{j} \eta_{j}$, then $\left\langle|H|^{1 / 2} \eta, \operatorname{sgn}(H)|H|^{1 / 2} \eta\right\rangle=e_{n}\|\eta\|$. It follows by the spectral theorem that there are at least $m+1$ eigenvalues in $\left(-\infty, e_{n}\right]$. Since $H$ has separated boundary conditions, its point spectrum is simple.

The second part of the proof of Theorem 2.1 also shows:

Corollary 2.4. Let $H$ be an SL operator bounded from below. If $\psi_{+}(E, \cdot)$ (resp. $\left.\psi_{-}(E, \cdot)\right)$ has $m$ zeros, then there are at least $m$ eigenvalues below $E$. In particular, $E$ below the spectrum of $H$ implies that $\psi_{ \pm}(E, \cdot)$ have no zeros.

3. Zeros of the Wronskian. Here we'll present the two aspects of zeros of the Wronskian which are critical for the two halves of our proofs (i.e., for showing $N_{0} \geq W_{0}$ and that $\left.N_{0} \leq W_{0}\right)$. First, the vanishing of the Wronskian lets us patch solutions together:

Proposition 3.1. Suppose that $\psi_{+, j}, \psi_{-} \in D_{\text {loc }}$ and that $\psi_{+, j}$ and $\tau \psi_{+, j}, j=1,2$ are in $L^{2}((c, b))$ and that $\psi_{-}$and $\tau \psi_{-}$are in $L^{2}((a, c))$ for all $c \in(a, b)$. Suppose, 
in addition, that $\psi_{+, j}, j=1,2$ satisfy the boundary condition defining $H$ at $b$ (i.e., $W\left(u, \psi_{+, j}\right)(c) \rightarrow 0$ as $c \uparrow b$ for all $\left.u \in D(H)\right)$ and similarly, that $\psi_{-}$satisfies the boundary condition at $a$. Then

(i) If $W\left(\psi_{+, 1}, \psi_{+, 2}\right)(c)=0$ and $\left(\psi_{+, 2}(c),\left(p \psi_{+, 2}^{\prime}\right)(c)\right) \neq(0,0)$, then there exists a $\gamma$ such that

$$
\eta=\chi_{[c, b)}\left(\psi_{+, 1}-\gamma \psi_{+, 2}\right) \in D(H)
$$

and

$$
H \eta=\chi_{[c, b)}\left(\tau \psi_{+, 1}-\gamma \tau \psi_{+, 2}\right)
$$

(ii) If $W\left(\psi_{+, 1}, \psi_{-}\right)(c)=0$ and $\left(\psi_{-}(c),\left(p \psi_{-}^{\prime}\right)(c)\right) \neq(0,0)$, then there is a $\gamma$ such that

$$
\eta=\gamma \chi_{(a, c]} \psi_{-}+\chi_{(c, b)} \psi_{+, 1} \in D(H)
$$

and

$$
H \eta=\gamma \chi_{(a, c]} \tau \psi_{-}+\chi_{(c, b)} \tau \psi_{+, 1}
$$

Proof. Clearly, $\eta$ and the right-hand sides of (3.1)/(3.2) lie in $L^{2}((a, b))$ and satisfy the boundary condition at $a$ and $b$, so it suffices to prove that $\eta$ and $p \eta^{\prime}$ are locally absolutely continuous on $(a, b)$.

In case (i), if $\psi_{+, 2}(c) \neq 0$, take $\gamma=-\psi_{+, 1}(c) / \psi_{+, 2}(c)$ and otherwise (i.e., if $\left.\psi_{+, 2}(c)=0\right)$ take $\gamma=-\left(p \psi_{+, 1}^{\prime}\right)(c) /\left(p \psi_{+, 2}^{\prime}\right)(c)$. In either case, $\eta$ and $p \eta^{\prime}$ are continuous at $c$. Case (ii) is similar.

The second aspect connects zeros of the Wronskian to Prüfer variables $\rho_{u}, \theta_{u}$ (for $u, p u^{\prime}$ absolutely continuous) defined by

$$
u(x)=\rho_{u}(x) \sin \left(\theta_{u}(x)\right), \quad\left(p u^{\prime}\right)(x)=\rho_{u}(x) \cos \left(\theta_{u}(x)\right)
$$

If $\left(u(x),\left(p u^{\prime}\right)(x)\right)$ is never $(0,0)$, then $\rho_{u}$ can be chosen positive and $\theta_{u}$ is uniquely determined once a value of $\theta_{u}\left(x_{0}\right)$ is chosen subject to the requirement $\theta_{u}$ continuous in $x$.

Notice that

$$
W\left(u_{1}, u_{2}\right)(x)=\rho_{u_{1}}(x) \rho_{u_{2}}(x) \sin \left(\theta_{u_{1}}(x)-\theta_{u_{2}}(x)\right) .
$$


Thus,

Proposition 3.2. Suppose $\left(u_{j}, p u_{j}^{\prime}\right), j=1,2$ are never $(0,0)$. Then $W\left(u_{1}, u_{2}\right)\left(x_{0}\right)$ is zero if and only if $\theta_{u_{1}}\left(x_{0}\right) \equiv \theta_{u_{2}}\left(x_{0}\right)(\bmod \pi)$.

In linking Prüfer variables to rotation numbers, an important role is played by the observation that because of

$$
u(x)=\int_{x_{0}}^{x} \frac{\rho_{u}(t) \cos \left(\theta_{u}(t)\right)}{p(t)} d t
$$

$\theta_{u}\left(x_{0}\right) \equiv 0(\bmod \pi)$ implies $\left[\theta_{u}(x)-\theta_{u}\left(x_{0}\right)\right] /\left(x-x_{0}\right)>0$ for $0<\left|x-x_{0}\right|$ sufficiently small and hence for all $0<\left|x-x_{0}\right|$ if $\left(u, p u^{\prime}\right) \neq(0,0)$. (In fact, suppose $x_{1} \neq x_{0}$ is the closest $x$ such that $\theta_{u}\left(x_{1}\right)=\theta_{u}\left(x_{0}\right)$, then apply the local result at $x_{1}$ to obtain a contradiction.) We summarize:

Proposition 3.3. If $\left(u, p u^{\prime}\right) \neq(0,0)$ then $\theta_{u}\left(x_{0}\right) \equiv 0(\bmod \pi)$ implies

$$
\left[\theta_{u}(x)-\theta_{u}\left(x_{0}\right)\right] /\left(x-x_{0}\right)>0
$$

for $x \neq x_{0}$. In particular, if $\theta_{u}(c) \in[0, \pi)$ and $u$ has $n$ zeros in $(c, d)$, then $\theta_{u}(d-\epsilon) \in$ $(n \pi,(n+1) \pi)$ for sufficiently small $\epsilon>0$.

In exactly the same way, we have

Proposition 3.4. Let $E_{1}<E_{2}$ and assume that $u_{1,2}$ solve $\tau u_{j}=E_{j} u_{j}, j=1,2$. Let $\Delta(x)=\theta_{u_{2}}(x)-\theta_{u_{1}}(x)$. Then $\Delta\left(x_{0}\right) \equiv 0(\bmod \pi)$ implies $\left(\Delta(x)-\Delta\left(x_{0}\right)\right) /\left(x-x_{0}\right)>$ 0 for $0<\left|x-x_{0}\right|$.

Proof. If $\Delta\left(x_{0}\right) \equiv 0(\bmod 2 \pi)$ and $\theta_{u_{2}}\left(x_{0}\right) \not \equiv 0(\bmod \pi)$, then

$$
\sin \left(\theta_{u_{2}}\left(x_{0}\right)\right) \sin \left(\theta_{u_{1}}\left(x_{0}\right)\right)>0
$$

so $u_{1}\left(x_{0}\right) u_{2}\left(x_{0}\right)>0$ for $0<\left|x-x_{0}\right|$ sufficiently small, and thus by (2.2), $\frac{d W}{d x}\left(x_{0}\right)>$ 0 for a.e. $x$ near $x_{0}$ and so $\Delta(x)$ is increasing. The same holds for $\Delta\left(x_{0}\right) \equiv \pi$ $(\bmod 2 \pi)$ and $\theta_{u_{2}}\left(x_{0}\right) \not \equiv 0(\bmod \pi)$.

If $\Delta\left(x_{0}\right) \equiv 0(\bmod 2 \pi)$ and $\theta_{u_{1}}\left(x_{0}\right) \equiv \theta_{u_{2}}\left(x_{0}\right) \equiv 0(\bmod \pi)$, then

$$
\left(p u_{1}^{\prime}\right)\left(x_{0}\right)\left(p u_{2}^{\prime}\right)\left(x_{0}\right)>0
$$

and so since $u\left(x_{0}\right)=\mathrm{V}\left(x_{0}\right)=0$, we see that it is still true that $\frac{d W}{d x}(x)>0$ a.e. for $0<\left|x-x_{0}\right|$ sufficiently small.

Remarks. (i) Suppose $r, p$ are continuous on $(a, b)$. If $\theta_{u_{1}}\left(x_{0}\right) \equiv 0(\bmod \pi)$ then $\theta_{u_{1}}(x)-\theta_{u_{1}}\left(x_{0}\right)=c_{0}\left(x-x_{0}\right)+o\left(x-x_{0}\right)$ with $c_{0}>0$. If $\Delta\left(x_{0}\right) \equiv 0(\bmod \pi)$ 
and $\theta_{u_{1}}\left(x_{0}\right) \not \equiv 0(\bmod \pi)$, then $\Delta(x)-\Delta\left(x_{0}\right)=c_{1}\left(x-x_{0}\right)+o\left(x-x_{0}\right)$ with $c_{1}>0$. If $\theta_{u_{1}}\left(x_{0}\right) \equiv 0 \equiv \Delta\left(x_{0}\right)(\bmod \pi)$, then $\left.\Delta(x)-\Delta\left(x_{0}\right)=c_{2}\left(x-x_{0}\right)^{3}+o\left(x-x_{0}\right)^{3}\right)$ with $c_{2}>0$. Either way, $\Delta$ increases through $x_{0}$. (In fact, $c_{0}=p\left(x_{0}\right)^{-1}, c_{1}=$ $\left(E_{2}-E_{1}\right) r\left(x_{0}\right) \sin ^{2}\left(\theta_{u_{1}}\left(x_{0}\right)\right)$ and $\left.c_{2}=\frac{1}{3} r\left(x_{0}\right) p\left(x_{0}\right)^{-2}\left(E_{2}-E_{1}\right)\right)$.

(ii) In other words, Propositions 3.3 and 3.4 say that the integer parts of $\theta_{u} / \pi$ and $\Delta_{u, \mathrm{v}} / \pi$ are increasing with respect to $x \in(a, b)$ (even though $\theta_{u}$ and $\Delta_{u, \mathrm{~V}}$ themselves are not necessarily monotone in $x$ ).

(iii) Let $E \in\left[E_{1}, E_{2}\right]$ and assume $\left[E_{1}, E_{2}\right]$ to be outside the essential spectrum of $H$. Then, for $x \in(a, b)$ fixed,

$$
\frac{d \theta_{\psi_{ \pm}}}{d E}(E, x)=-\frac{\int_{x}^{\ell_{ \pm}} \psi_{ \pm}(E, t)^{2} d t}{\rho_{\psi_{ \pm}}(E, x)}
$$

proves that $\mp \theta_{\psi_{ \pm}}(E, x)$ is strictly increasing with respect to $E$. In fact, from Proposition 2.3 one infers

$$
W\left(\psi_{ \pm}(E), \psi_{ \pm}(\tilde{E})\right)(x)=(\tilde{E}-E) \int_{x}^{\ell_{ \pm}} \psi_{ \pm}(E, t) \psi_{ \pm}(\tilde{E}, t) d t
$$

and using this to evaluate the $\operatorname{limit}_{\tilde{E} \rightarrow E} W\left(\psi_{ \pm}(E),\left(\psi_{ \pm}(E)-\psi_{ \pm}(\tilde{E})\right) /(E-\right.$ $\tilde{E})(x)$, one obtains

$$
W\left(\psi_{ \pm}(E), \frac{d \psi_{ \pm}}{d E}(E)\right)(x)=\int_{x}^{\ell_{ \pm}} \psi_{ \pm}(E, t)^{2} d t
$$

Inserting Prüfer variables completes the proof of (3.3).

4. The hare and the tortoise ( $N_{0} \leq W_{0}$ in the regular case). Our goal in this section is to prove Theorem 1.5 in the regular case with separated boundary conditions, that is,

Proposition 4.1. Let $H$ be a regular $S L$ operator and suppose $E_{1}<E_{2}$. Then

$$
W_{0}\left(\psi_{-}\left(E_{1}\right), \psi_{+}\left(E_{2}\right)\right) \geq N_{0}\left(E_{1}, E_{2}\right) .
$$

The proof will use Prüfer angles. As a warm-up, let us prove equality in the case that $H$ has $u(a)=u(b)=0$ boundary conditions and that $E_{1,2}$ are not eigenvalues. Let $\theta_{\psi_{ \pm}}(E, x)$ be the Prüfer angles for $\psi_{ \pm}(E, x)$, normalized such that $\theta_{\psi_{ \pm}}(E, a) \in[0, \pi)$. Since $\psi_{-}\left(E_{1}\right)$ satisfies the boundary condition at $a, \theta_{\psi_{-}}\left(E_{1}, a\right)=0$ and since $E_{2}$ is not an eigenvalue, $\theta_{\psi_{+}}\left(E_{2}, a\right)>0$. If there are $m$ eigenvalues below $E_{1}$ and $n_{0}+m$ below $E_{2}$, then, by standard oscillation 
theory (essentially Proposition 3.3), $\theta_{\psi_{-}}\left(E_{1}, b\right) \in(m \pi,(m+1) \pi)$ and $\theta_{\psi_{+}}\left(E_{2}, b\right)=$ $\left(n_{0}+m+1\right) \pi$. Let $\Gamma_{ \pm}(E, x) \equiv \theta_{\psi_{ \pm}}(E, x)(\bmod \pi)$, that is, $\Gamma_{ \pm}(E, x) \in[0, \pi)$ and $\Gamma_{ \pm}-\theta_{\psi_{ \pm}} \in \mathbb{Z} \pi$.

Borrow a leaf from Aesop. Think of $\Gamma_{-}\left(E_{1}\right)$ as a tortoise and $\Gamma_{+}\left(E_{2}\right)$ as a hare racing on a track of size $\pi$ with 0 as the start and $\pi$ as the finish. Every time either runs through the finish, the race starts all over. Neither has to run only in the forward direction (i.e., $\theta_{\psi_{ \pm}}$may not be monotone w.r.t. $x$ ) but they can't run in the wrong direction back through the start (i.e., Proposition 3.3 holds).

What makes $\Gamma_{+}\left(E_{2}\right)$ the hare to $\Gamma_{-}\left(E_{1}\right)$ 's tortoise is that $\Gamma_{+}\left(E_{2}\right)$ can only overtake $\Gamma_{-}\left(E_{1}\right)$, not the other way around (i.e., Proposition 3.4 holds). Since $\Gamma_{-}\left(E_{1}, a\right)=0$ and $\Gamma_{+}\left(E_{2}, a\right)>0$, the hare starts out ahead of the tortoise. Since $\Gamma_{-}\left(E_{1}, c\right)<\pi$ but $\Gamma_{+}\left(E_{1}, c\right) \nearrow \pi$ as $c \nearrow b$, the hare also ends up ahead (unlike in Aesop!).

Clearly, the number of times the hare crosses the finish line is the sum of the number of times the tortoise does, plus the number of times the hare "laps," that is, passes the tortoise. Thus,

$$
n_{0}+m=m+W_{0}\left(\psi_{-}\left(E_{1}\right), \psi_{+}\left(E_{2}\right)\right)
$$

so $W_{0}\left(\psi_{-}\left(E_{1}\right), \psi_{+}\left(E_{2}\right)\right)=n_{0}$ in the Dirichlet case.

This picture also explains why it can happen that

$$
W_{0}\left(\psi_{-}\left(E_{1}\right), \psi_{-}\left(E_{2}\right)\right)=n_{0}-1
$$

For in this case, $\theta_{\psi_{-}}\left(E_{1}, a\right)=\theta_{\psi_{-}}\left(E_{2}, a\right)=0$. The hare and tortoise start out together, so for $x=a+\epsilon$, the hare is slightly ahead. If at $b, \Gamma_{-}\left(E_{1}, b\right)>\Gamma_{-}\left(E_{2}, b\right)$, then the tortoise à la Aesop wins the races; thus the hare has lapped the tortoise one time too few, that is,

$$
n_{0}+m-1=m+W_{0}\left(\psi_{-}\left(E_{1}\right), \psi_{-}\left(E_{2}\right)\right)
$$

and so

$$
W_{0}=n_{0}-1
$$

Suppose $E_{1}<e<E_{2}$ with $e$ an eigenvalue. As $E_{2} \searrow e, \Gamma_{-}\left(E_{2}, b\right) \searrow 0$ as $E_{1} \nearrow e, \Gamma_{-}\left(E_{1}, b\right) \nearrow \pi$. Thus for $E_{2}-E_{1}$ sufficiently small, $\Gamma_{-}\left(E_{2}, b\right)<$ $\Gamma_{-}\left(E_{1}, b\right)$ and (4.2) holds.

Now we turn to the proof of Proposition 4.1 in the general case (assuming $H$ to be a regular SL operator for the rest of this section).

LeMmA 4.2. Let $u_{1,2}$ be eigenfunctions of $H$ with eigenvalues $E_{1}<E_{2}$. Let $\ell$ be the number of eigenvalues of $H$ in $\left(E_{1}, E_{2}\right)$. Then $W\left(u_{1}, u_{2}\right)(x)$ has exactly $\ell$ zeros in $(a, b)$. 
Proof. Suppose $u_{1}$ is the $k$ th eigenfunction. By Theorem 2.1, $u_{1}$ has $k-1$ zeros and $u_{2}$ has $k+\ell$ zeros in $(a, b)$. Moreover, $\Gamma_{-}\left(E_{1}, a\right)=\Gamma_{+}\left(E_{2}, a\right), \Gamma_{-}\left(E_{1}, b\right)=$ $\Gamma_{+}\left(E_{2}, b\right)$ so $\Gamma_{-}\left(E_{1}, a+\epsilon\right)<\Gamma_{+}\left(E_{2}, a+\epsilon\right), \Gamma_{+}\left(E_{2}, b-\epsilon\right)<\Gamma_{-}\left(E_{1}, b-\epsilon\right)$. So the hare starts slightly ahead and ends slightly behind and so it laps one less time than the difference of the number of zeros, that is, $W_{0}\left(u_{1}, u_{2}\right)=(\ell+1)-1=\ell$.

Lemma 4.3. Let $E_{1} \leq E_{2}$ be eigenvalues of $H$ and suppose $\left[E_{1}, E_{2}\right]$ has $\ell$ eigenvalues. Then for $\epsilon \geq 0$ sufficiently small, $W_{0}\left(\psi_{-}\left(E_{1}-\epsilon\right), \psi_{+}\left(E_{2}+\epsilon\right)\right)=\ell$.

Remark. Since $\left(E_{1}, E_{2}\right)$ has $\ell-2$ eigenvalues, Lemma 4.2 says that $W\left(\psi_{-}\left(E_{1}\right), \psi_{+}\left(E_{2}\right)\right)(x)$ has $\ell-2$ zeros in $(a, b)$ and clearly it has zeros at $a$ and $b$. Essentially, Lemma 4.3 says that replacing $E_{1}$ by $E_{1}-\epsilon$ and $E_{2}$ by $E_{2}+\epsilon$ moves the zeros at $a, b$ inside $(a, b)$ to give $\ell-2+2=\ell$ zeros.

Proof. Suppose first $E_{1}<E_{2}$. Compare the tortoises associated to $\psi_{-}\left(E_{1}-\epsilon\right)$ and $\psi_{-}\left(E_{1}\right)$. The first starts out at $x=a$ in the same position as the second (i.e., $\left.\Gamma_{-}\left(E_{1}-\epsilon, a\right)=\Gamma_{-}\left(E_{1}, a\right)\right)$, which means it must end slightly behind, that is, $\Gamma_{-}\left(E_{1}-\epsilon, b\right)<\Gamma_{-}\left(E_{1}, b\right)$. Similarly, since the faster hare for energy $E_{2}+\epsilon$ ends up where the hare of energy $E_{2}$ does (i.e, $\left.\Gamma_{+}\left(E_{2}+\epsilon, b\right)=\Gamma_{+}\left(E_{2}, b\right)\right)$, it must start out slightly farther back, that is, $\Gamma_{+}\left(E_{2}+\epsilon, a\right)<\Gamma_{+}\left(E_{2}, a\right)$. Thus $W\left(\psi_{-}\left(E_{1}-\right.\right.$ $\left.\epsilon), \psi_{+}\left(E_{2}+\epsilon\right)\right)(x)$ picks up two zeros over the $\ell-2$ that $W\left(\psi_{-}\left(E_{1}\right), \psi_{+}\left(E_{2}\right)\right)(x)$ has.

If $E_{2}=E_{1} \equiv E$, the $\Gamma_{+}$for $\psi_{+}(E+\epsilon)$ starts out slightly behind the one for $\psi_{+}(E)$ and ends up slightly ahead of the $\Gamma_{-}$for $\psi_{-}(E-\epsilon)$, so there has to be one crossing, that is, $W_{0}\left(\psi_{-}(E-\epsilon), \psi_{+}(E+\epsilon)\right)=1$.

LEMMA 4.4. If $E_{3}<E_{4}<E$ and $u$ is any solution of $\tau u=E u$, then

$$
W_{0}\left(\psi_{-}\left(E_{3}\right), u\right) \geq W_{0}\left(\psi_{-}\left(E_{4}\right), u\right)
$$

Similarly, if $E_{3}>E_{4}>E$ and $u$ is any solution of $\tau u=E u$, then (4.3) holds.

Proof. In the first case, think of $u$ as defining a hare and $\psi_{-}\left(E_{j}\right), j=3,4$ as defining tortoises. The $E_{3}$ and $E_{4}$ tortoises start out at the same place and the $E_{3}$ tortoise runs "faster" in that it is always ahead after the start. Clearly, the hare will pass the slower tortoise at least as often as the faster one.

In the second case, there are two hares (defined by $\left.\psi_{-}\left(E_{j}\right), j=3,4\right)$, which start out at the same place, and one tortoise (defined by $u$ ) and it is clear that the faster hare (given by $\psi_{-}\left(E_{3}\right)$ ) has to pass the tortoise at least as often as the slower one.

LEMMA 4.5. Lemma 4.4 remains true if every $\psi_{-}$is replaced by a $\psi_{+}$.

Proof. Reflect at some point $c \in(a, b)$ implying an interchange of $\psi_{+}$ and $\psi_{-}$. 
Proof of Proposition 4.1. If $N_{0}=0$, there is nothing to prove. If $N_{0} \geq 1$, let $\operatorname{spec}(H) \cap\left(E_{1}, E_{2}\right)=\left\{e_{m}\right\}_{m \in M}$ and let $e_{s} \leq e_{\ell}$ be the smallest and largest of the $e_{m}$ 's. Thus, $N_{0}$ is the number of eigenvalues in $\left[e_{s}, e_{\ell}\right]$ and so

$$
N_{0}=W_{0}\left(\psi_{-}\left(e_{s}-\epsilon\right), \psi_{+}\left(e_{\ell}+\epsilon\right)\right)
$$

by Lemma 4.3. By Lemma 4.4,

$$
W_{0}\left(\psi_{-}\left(e_{s}-\epsilon\right), \psi_{+}\left(e_{\ell}+\epsilon\right)\right) \leq W_{0}\left(\psi_{-}\left(E_{1}\right), \psi_{+}\left(e_{\ell}+\epsilon\right)\right),
$$

and then by Lemma 4.5 , this is no larger than $W_{0}\left(\psi_{-}\left(E_{1}\right), \psi_{+}\left(E_{2}\right)\right)$.

5. Strong limits $\left(N_{0} \leq W_{0}\right.$ in the general case). Using the approach of Weidmann ([22], chapter 14) to control some limits, we prove Theorem 1.5 in this section. Fix functions $u_{1}, u_{2} \in D_{\text {loc }}$. Pick $c_{n} \downarrow a, d_{n} \uparrow b$. Define $\tilde{H}_{n}$ on $L^{2}\left(\left(c_{n}, d_{n}\right) ; r d x\right)$ by imposing the following boundary conditions on $\eta \in D\left(\tilde{H}_{n}\right)$

$$
W\left(u_{1}, \eta\right)\left(c_{n}\right)=0=W\left(u_{2}, \eta\right)\left(d_{n}\right) .
$$

On $L^{2}((a, b) ; r d x)=L^{2}\left(\left(a, c_{n}\right) ; r d x\right) \oplus L^{2}\left(\left(c_{n}, d_{n}\right) ; r d x\right) \oplus L^{2}\left(\left(d_{n}, b\right) ; r d x\right)$ take $H_{n}=$ $\alpha \mathbb{I} \oplus \tilde{H}_{n} \oplus \alpha \mathbb{I}$ with $\alpha$ a fixed real constant. Then Weidmann proves:

Lemma 5.1. Suppose that either $H$ is limit point at a or that $u_{1}$ is an $\psi_{-}(E, x)$ for some $E$ and similarly, that either $H$ is limit point at $b$ or $u_{2}$ is an $\psi_{+}\left(E^{\prime}, x\right)$ for some $E^{\prime}$. Then $H_{n}$ converges to $H$ in strong resolvent sense as $n \rightarrow \infty$.

The idea of Weidmann's proof is that it suffices to find a core $D_{0}$ of $H$ such that for every $\eta \in D_{0}$ there exists an $n_{0} \in \mathbb{N}$ with $\eta \in D_{0}$ for $n \geq n_{0}$ and $H_{n} \eta \rightarrow H \eta$ as $n$ tends to infinity (see [21], theorem 9.16(i)). If $H$ is limit point at both ends, take $\eta \in D_{0} \equiv\left\{u \in D_{\text {loc }} \mid \operatorname{supp}(u)\right.$ compact in $\left.(a, b)\right\}$. Otherwise, pick $\tilde{u}_{1}, \tilde{u}_{2} \in D(H)$ with $\tilde{u}_{2}=u_{2}$ near $b$ and $\tilde{u}_{2}=0$ near $a$ and with $\tilde{u}_{1}=u_{1}$ near $a$ and $\tilde{u}_{1}=0$ near $b$. Then pick $\eta \in D_{0}+\operatorname{span}\left[\tilde{u}_{1}, \tilde{u}_{2}\right]$ which one can show is a core for $H$ ([22], chapter 14).

Secondly we note:

Lemma 5.2. Let $A_{n} \rightarrow A$ in strong resolvent sense as $n \rightarrow \infty$. Then

$$
\operatorname{dim} \operatorname{Ran} P_{\left(E_{1}, E_{2}\right)}(A) \leq \underset{n \rightarrow \infty}{\lim } \operatorname{dim} \operatorname{Ran} P_{\left(E_{1}, E_{2}\right)}\left(A_{n}\right)
$$

Proof. Fix $m \leq \operatorname{dim} \operatorname{Ran} P_{\left(E_{1}, E_{2}\right)}(A)$ with $m<\infty$. We'll prove $m \leq$ RHS of (5.2). Suppose first that $\left(E_{1}, E_{2}\right)$ aren't eigenvalues of $A$. Then by theorem VIII.24 of [17], $P_{\left(E_{1}, E_{2}\right)}\left(A_{n}\right) \rightarrow P_{\left(E_{1}, E_{2}\right)}(A)$ strongly as $n \rightarrow \infty$. Picking orthonormal 
$\varphi_{1}, \ldots, \varphi_{m}$ in $\operatorname{Ran} P_{\left(E_{1}, E_{2}\right)}(A)$, we see that

$$
\varliminf_{n \rightarrow \infty} \operatorname{Tr}\left(P_{\left(E_{1}, E_{2}\right)}\left(A_{n}\right)\right) \geq \underset{n \rightarrow \infty}{\lim _{j}} \sum_{j}\left\langle\varphi_{j}, P_{\left(E_{1}, E_{2}\right)}\left(A_{n}\right) \varphi_{j}\right\rangle=m
$$

as required.

If $E_{1,2}$ are arbitrary, we can always find a $\delta>0$ such that $E_{1}+\delta, E_{2}-\delta$ are not eigenvalues of $A$ and such that $\operatorname{dim} \operatorname{Ran} P_{\left(E_{1}+\delta, E_{2}-\delta\right)}(A) \geq m$. Thus,

$$
\varliminf_{n \rightarrow \infty} \operatorname{dim} \operatorname{Ran} P_{\left(E_{1}, E_{2}\right)}\left(A_{n}\right) \geq \underset{n \rightarrow \infty}{\lim _{n \rightarrow \infty}} \operatorname{dim} \operatorname{Ran} P_{\left(E_{1}+\delta, E_{2}-\delta\right)}\left(A_{n}\right) \geq m .
$$

Proof of Theorem 1.5. Let $c_{n} \downarrow a, d_{n} \uparrow b$ and $H_{n}$ be as in Lemma 5.1 with $\alpha \notin\left[E_{1}, E_{2}\right]$. Proposition 4.1 implies $W_{0}\left(u_{1}, u_{2}\right) \geq \operatorname{dim} \operatorname{Ran} P_{\left(E_{1}, E_{2}\right)}\left(\tilde{H}_{n}\right)=$ $\operatorname{dim} \operatorname{Ran} P_{\left(E_{1}, E_{2}\right)}\left(H_{n}\right)$ since $\alpha \notin\left[E_{1}, E_{2}\right]$. Thus by Lemmas 5.1 and 5.2,

$$
W_{0}\left(u_{1}, u_{2}\right) \geq \operatorname{dim} \operatorname{Ran} P_{\left(E_{1}, E_{2}\right)}(H)
$$

as was to be proven.

6. A variational argument $\left(N_{0} \geq W_{0}\right)$. In this section, we'll prove Theorem 1.6. Let $E_{1}<E_{2}$. Suppose first that $u_{1}=\psi_{-}\left(E_{1}\right)$ and $u_{2}=\psi_{+}\left(E_{2}\right)$. Let $x_{1}, \ldots, x_{m}$ be zeros of $W\left(u_{1}, u_{2}\right)(x)$. We'll prove that $\operatorname{dim} P_{\left(E_{1}, E_{2}\right)}(H) \geq m$. If $W_{0}\left(u_{1}, u_{2}\right)=m$, this proves (1.8). If $W_{0}=\infty$, we can take $m$ arbitrarily large, and again (1.8) holds. Define

$$
\eta_{j}(x)=\left\{\begin{array}{ll}
u_{1}(x), & x \leq x_{j} \\
\gamma_{j} u_{2}(x), & x \geq x_{j}
\end{array}, \quad 1 \leq j \leq m,\right.
$$

where $\gamma_{j}$ is defined such that $\eta_{j} \in D(H)$ by Proposition 3.1. Let

$$
\tilde{\eta}_{j}(x)=\left\{\begin{array}{ll}
u_{1}(x), & x \leq x_{j} \\
-\gamma_{j} u_{2}(x), & x>x_{j}
\end{array}, \quad 1 \leq j \leq m .\right.
$$

If $E_{1}$ is an eigenvalue of $H$, we define in addition $\eta_{0}=u_{2}=-\tilde{\eta}_{0}, x_{0}=a$ and if $E_{2}$ is an eigenvalue of $H, \eta_{m+1}=u_{1}=\tilde{\eta}_{m+1}, x_{m+1}=b$.

LeMma 6.1. $\left\langle\eta_{j}, \eta_{k}\right\rangle=\left\langle\tilde{\eta}_{j}, \tilde{\eta}_{k}\right\rangle$ for all $j, k$ where $\langle\cdot, \cdot\rangle$ is the $L^{2}((a, b) ; r d x)$ inner product.

Proof. Let $j<k$. This just says that $\int_{x_{j}}^{x_{k}} u_{1}(x) u_{2}(x) r(x) d x=0$. But by (2.1), this integral is $\left(E_{1}-E_{2}\right)^{-1}\left[W\left(u_{1}, u_{2}\right)\left(x_{k}\right)-W\left(u_{1}, u_{2}\right)\left(x_{j}\right)\right]=0$ since $W\left(u_{1}, u_{2}\right)(\cdot)$ vanishes at $x_{\ell}$ respectively in the limit (if $x_{\ell}=a, b$ ) by Proposition 1.1. 
Notice that by (3.2),

$$
\left(H-\frac{E_{2}+E_{1}}{2}\right) \eta_{j}=\left(\frac{E_{2}-E_{1}}{2}\right) \tilde{\eta}_{j}
$$

This result and Lemma 6.1 imply

LEMMA 6.2. If $\eta$ is in the span of the $\eta_{j}$, then

$$
\left\|\left(H-\frac{E_{2}+E_{1}}{2}\right) \eta\right\|=\frac{\left|E_{2}-E_{1}\right|}{2}\|\eta\|
$$

Thus, $\operatorname{dim} \operatorname{Ran} P_{\left[E_{1}, E_{2}\right]}(H) \geq \operatorname{dim}\left(\operatorname{span}\left(\left\{\eta_{j}\right\}\right)\right)$. But $u_{1}$ and $u_{2}$ are independent on each interval (since their Wronskian is nonconstant) and so the $\eta_{j}$ are linearly independent. This proves Theorem 1.6 in the $\psi_{-}\left(E_{1}\right), \psi_{+}\left(E_{2}\right)$ case.

The case $u_{1}=\psi_{-}\left(E_{1}\right), u_{2}=\psi_{-}\left(E_{2}\right)$ is similar. We define

$$
\eta_{j}(x)=\left\{\begin{array}{ll}
u_{1}(x)-\gamma_{j} u_{2}(x), & x \leq x_{j} \\
0, & x \geq x_{j}
\end{array}, \quad 1 \leq j \leq m\right.
$$

and

$$
\tilde{\eta}_{j}(x)=\left\{\begin{array}{ll}
u_{1}(x)+\gamma_{j} u_{2}(x), & x \leq x_{j} \\
0, & x>x_{j}
\end{array}, \quad 1 \leq j \leq m .\right.
$$

If $E_{1}$ is an eigenvalue of $H$, we define in addition $\eta_{0}=u_{2}=-\tilde{\eta}_{0}, x_{0}=b$ and if $E_{2}$ is an eigenvalue of $H, \eta_{m+1}=u_{1}=\tilde{\eta}_{m+1}, x_{m+1}=b$. Again, $\eta_{j}$ 's are linearly independent by considering their supports. To prove the analog of Lemma 6.1, we need

$$
\int_{a}^{x_{j}} u_{1}(x) u_{2}(x) r(x) d x=0 .
$$

But by (2.1), this integral is

$$
\lim _{c \downarrow a}\left(E_{1}-E_{2}\right)^{-1}\left[W\left(u_{1}, u_{2}\right)\left(x_{j}\right)-W\left(u_{1}, u_{2}\right)(c)\right] .
$$

By hypothesis, $W\left(u_{1}, u_{2}\right)\left(x_{j}\right)=0$ and since $u_{1}$ and $u_{2}$ satisfy the boundary condition at $a, W\left(u_{1}, u_{2}\right)(c) \rightarrow 0$ as $c \downarrow a$ by Proposition 1.1. The cases $u_{1}=\psi_{+}\left(E_{1}\right)$, $u_{2}=\psi_{ \pm}\left(E_{2}\right)$ can be obtained by reflection.

7. Extensions, comments, and examples. The following includes Theorem 1.7: 
THEOREM 7.1. Let $E_{1} \neq E_{2}$. Let $\tau u_{j}=E_{j} u_{j}, j=1,2, \tau \mathrm{V}_{2}=E_{2} \mathrm{~V}_{2}$ with $u_{2}$ linearly independent of $\mathrm{v}_{2}$. Then the zeros of $W\left(u_{1}, u_{2}\right)$ interlace the zeros of $W\left(u_{1}, \mathrm{v}_{2}\right)$ and vice versa (in the sense that there is exactly one zero of one function in between two zeros of the other). In particular, $\left|W_{0}\left(u_{1}, u_{2}\right)-W_{0}\left(u_{1}, \mathrm{v}_{2}\right)\right| \leq 1$.

Proof. We'll suppose $E_{1}<E_{2}$. A similar argument works if $E_{2}<E_{1}$. In the language of Section $4, \Gamma_{u_{1}}$ represents the tortoise and $\Gamma_{u_{2}}, \Gamma_{\mathrm{V}_{2}}$ are two hares. Since $W\left(u_{2}, \mathrm{v}_{2}\right)$ is a nonzero constant, one hare always stays ahead of the other. It follows that if the hare $\Gamma_{u_{1}}$ crosses the tortoise $\Gamma_{u_{2}}$ at $x_{1}$ and $x_{2}, x_{1}<x_{2}$, the hare $\Gamma_{\mathrm{V}_{2}}$ must cross it at some point in $\left(x_{1}, x_{2}\right)$.

By applying this theorem twice, we conclude

THEOREM 7.2. Let $E_{1} \neq E_{2}$. Let $u_{1}, u_{2}, \mathrm{v}_{1}, \mathrm{v}_{2}$ be the linearly independent functions with $\tau u_{j}=E_{j} u_{j}$ and $\tau \mathrm{V}_{j}=E_{j} \mathrm{~V}_{j}$. Then

$$
\left|W_{0}\left(u_{1}, u_{2}\right)-W_{0}\left(\mathrm{v}_{1}, \mathrm{v}_{2}\right)\right| \leq 2
$$

THEOREM 7.3. If $\operatorname{dim} \operatorname{Ran} P_{\left(E_{1}, E_{2}\right)}(H)=\infty$, then $W_{0}\left(u_{1}, u_{2}\right)=\infty$ for any $u_{1}$ and $u_{2}$ satisfying $\tau u_{j}=E_{j} u_{j}, j=1,2$.

Proof. Firstly, if $W_{0}\left(u_{1}, u_{2}\right)=\infty$ for one pair $u_{1,2}$ this is true for any pair by Theorem 7.2. Secondly, pick $u_{1,2}$ such that the operator $H_{n}$ of Lemma 5.1 converges to $H$ in a strong resolvent sense as $n \rightarrow \infty$. Hence by Theorem 1.3 (applied to $\tilde{H}_{n}$ defined before Lemma 5.1) and Lemma 5.2 the number of zeros of the Wronskian in $\left(c_{n}, d_{n}\right)$ must go to infinity as $n \rightarrow \infty$.

Example 1. Let us take $p=r=1, q=0$ with $[a, b]=[0,1]$ and Neumann boundary conditions $u^{\prime}(0)=u^{\prime}(1)=0$. Let $E_{1}=-k_{1}^{2}, E_{2}=k_{2}^{2}$, and $u_{1}(x)=$ $\psi_{-}\left(E_{1}, x\right), u_{2}(x)=\psi_{-}\left(E_{2}, x\right)$. Then $u_{1}(x)=\cosh \left(k_{1} x\right), u_{2}(x)=\cos \left(k_{2} x\right)$, and

$$
W\left(u_{1}, u_{2}\right)(x)=-k_{2} \cosh \left(k_{1} x\right) \sin \left(k_{2} x\right)-k_{1} \sinh \left(k_{1} x\right) \cos \left(k_{2} x\right)
$$

has no zero in $[0,1]$ if $0<k_{1}, 0<k_{2}<\frac{\pi}{2}$. Thus, while $N_{0}=1, W_{0}=0$, so we see that $W_{0}=N_{0}-1$, that is, (1.7) in Theorem 1.4 can happen if the boundary conditions hold on the same side (note that the problem is limit circle at $b=1$ as it must be, given Theorem 1.4). This result is not surprising since $W\left(u_{1}, u_{2}\right)$ contains no information about the boundary condition at $b$.

Example 2. Again $p=r=1, q=0$. Take $[a, b]=[-1,1]$. Consider the two sets of boundary conditions

$$
\begin{aligned}
& u( \pm 1)=0 \\
& u( \pm 1)= \pm u^{\prime}( \pm 1)
\end{aligned}
$$


with corresponding operators $H_{1}$ and $H_{2}$. The lowest eigenvalue of $H_{1}$ is $\frac{1}{4} \pi^{2} . H_{2}$ has 0 as an eigenvalue with eigenvector $\varphi(x)=x . H_{2}$ has the lowest eigenvalue at $\alpha$ where $\alpha$ satisfies $\sqrt{-\alpha} \tanh \sqrt{-\alpha}=1$ (i.e., $\alpha \sim-1.44$ ). Let $E_{1}=-2$, $E_{2}=0.5$, and $-u_{j}^{\prime \prime}=E_{j} u_{j},-\mathrm{v}_{j}^{\prime \prime}=E_{j} \mathrm{v}_{j}, j=1,2$, with $u_{2}(1)=\mathrm{v}_{1}(-1)=0$ and $\mathrm{V}_{2}(1)-\mathrm{v}_{2}^{\prime}(1)=u_{1}(0)+u_{1}^{\prime}(0)=0$. Since $H_{2}$ has two eigenvalues in $\left(E_{1}, E_{2}\right)$ and $H_{1}$ has none, we see by Theorem 1.3 that $W_{0}\left(\mathrm{v}_{1}, u_{2}\right)=0, W_{0}\left(u_{1}, \mathrm{v}_{2}\right)=2$, and thus any of $0, \pm 1, \pm 2$ can occur in Theorem 7.2.

THeOrem 7.4. Let $E_{1}<E_{2}$. Let $\tau u_{j}=E_{j} u_{j}, j=1$, 2. If $a<x_{0}<x_{1}<b$ are zeros of $u_{1}$ or of $W\left(u_{1}, u_{2}\right)(\cdot)$, then the number of zeros of $u_{2}$ inside $\left(x_{0}, x_{1}\right)$ equals the number of zeros of $W\left(u_{1}, u_{2}\right)(\cdot)$ inside $\left(x_{0}, x_{1}\right)$ plus the number of zeros of $u_{1}$ inside $\left(x_{0}, x_{1}\right)$ plus one.

Proof. Let $\Gamma_{u_{1}}$ be the tortoise and $\Gamma_{u_{2}}$ the hare. $\Gamma_{u_{2}}$ starts out ahead or equal and the number of times $\Gamma_{u_{2}}$ laps (inside $\left.\left(x_{0}, x_{1}\right)\right)$ is equal to $W_{0}\left(u_{1}, u_{2}\right)$. Since $\Gamma_{u_{2}}$ ends up slightly ahead (i.e., $\Gamma_{u_{2}}\left(E_{2}, b-\epsilon\right)>\Gamma_{u_{1}}\left(E_{1}, b-\epsilon\right)$ ), the number of zeros of $u_{2}$ equals the number of laps plus the number of zeros of $u_{1}$ plus one.

The following result is of special interest in connection with the problem of whether the total number of eigenvalues of $H$ in one of its essential spectral gaps is finite or infinite. In particular, the energies $E_{1}, E_{2}$ in Theorem 7.5 below may lie in the essential spectrum of $H$. For this purpose we consider an auxiliary Dirichlet operator $H_{x_{0}}^{D}, x_{0} \in(a, b)$ associated with $H . H_{x_{0}}^{D}$ is obtained by taking the direct sum of the restrictions $H_{x_{0}, \pm}^{D}$ of $H$ to $\left(a, x_{0}\right)$, respectively $\left(x_{0}, b\right)$, with a Dirichlet boundary condition at $x_{0}$. We emphasize that the Dirichlet boundary conditions can be replaced by boundary conditions of the type $\lim _{\epsilon \downarrow 0}\left[u^{\prime}\left(x_{0} \pm \epsilon\right)+\beta u\left(x_{0} \pm \epsilon\right)\right]=$ $0, \beta \in \mathbb{R}$.

THEOREM 7.5. Let $E_{1}<E_{2}$. Let $\tau u_{j}=E_{j} u_{j}, \tau s_{j}=E_{j} s_{j}$, and $s_{j}\left(E_{j}, x_{0}\right)=0$, $j=1,2$. Then we have

(i) $\operatorname{dim} \operatorname{Ran} P_{\left(E_{1}, E_{2}\right)}(H)<\infty$ if and only if $W_{0}\left(u_{1}, u_{2}\right)<\infty$.

(ii) $\operatorname{dim} \operatorname{Ran} P_{\left(E_{1}, E_{2}\right)}(H)-1 \leq \operatorname{dim} \operatorname{Ran} P_{\left(E_{1}, E_{2}\right)}\left(H_{x_{0}}^{D}\right) \leq \operatorname{dim} \operatorname{Ran} P_{\left(E_{1}, E_{2}\right)}(H)$ +2 .

(iii) $\quad W_{0}\left(s_{1}, s_{2}\right)-1 \leq \operatorname{dim} \operatorname{Ran} P_{\left(E_{1}, E_{2}\right)}\left(H_{x_{0}}^{D}\right) \leq W_{0}\left(s_{1}, s_{2}\right)+1$.

Proof. Items (ii), (iii), and Theorem 7.2 imply (i). If the essential spectrum of $H$ and $\left(E_{1}, E_{2}\right)$ are not disjoint, (ii) is clear. Otherwise, if the essential spectrum of $H$ and $\left(E_{1}, E_{2}\right)$ are disjoint, a standard rank-one perturbation argument, combined with the strict monotonicity of the Green's function $G\left(E, x_{0}, x_{0}\right)$ of $H$ with respect to $E$ in essential spectral gaps of $H$, applies. For (iii) it suffices to prove

$$
W_{0, \pm}\left(s_{1}, s_{2}\right) \leq \operatorname{dim} \operatorname{Ran} P_{\left(E_{1}, E_{2}\right)}\left(H_{x_{0}, \pm}^{D}\right) \leq W_{0, \pm}\left(s_{1}, s_{2}\right)+1 \text {, }
$$

where $W_{0, \pm}\left(s_{1}, s_{2}\right)$ abbreviates the number of zeros of the Wronskian $W\left(s_{1}, s_{2}\right)$ 
inside $\left(x_{0}, b\right)$, respectively $\left(a, x_{0}\right)$. But this is immediate from Theorems 1.5 and 1.6 .

Next we want to see how Theorem 1.2 (and hence Hartman's theorem [10]) follows from Theorem 1.4. We start by assuming $\tau$ to be oscillatory at $E_{2}$ near $b$. By Theorem 1.4, $W_{0}\left(u_{1}, u_{2}\right)=N_{0}$ since $H$ in Theorem 1.2 is assumed to be limit point at $b$, so we need only show that $W_{0}\left(u_{1}, u_{2}\right)=\frac{\lim }{c \uparrow b} N(c)$ in order to prove (1.5a). Suppose first that $W_{0}\left(u_{1}, u_{2}\right)=m<\infty$. Since $\tau$ is non-oscillatory at $E_{2}$ near $a$ we can pick $x_{0}$ such that $u_{2}$ and $W\left(u_{1}, u_{2}\right)$ have no zeros in $\left(a, x_{0}\right]$. Hence we can assume without loss of generality that $\theta_{u_{1}}\left(x_{0}\right)=\theta_{0} \in(0, \pi)$ and $\theta_{u_{2}}\left(x_{0}\right) \in\left(\theta_{0}, \pi\right)$. Let $x_{m}$ be the last zero of $W\left(u_{1}, u_{2}\right)(x)$ (set $m=0$ and skip equation (7.1) if there are no zeros). At $x_{m}$,

$$
\theta_{u_{2}}\left(x_{m}\right)=\theta_{u_{1}}\left(x_{m}\right)+m \pi
$$

and then

$$
\Gamma_{u_{2}}\left(x_{m}+\epsilon\right)>\Gamma_{u_{1}}\left(x_{m}+\epsilon\right)
$$

Let $N_{u_{j}}(x)$ be the number of zeros of $u_{j}, j=1,2$ in $(a, x)$. By (7.1) and Proposition 3.3,

$$
N_{u_{2}}\left(x_{m}\right)=N_{u_{1}}\left(x_{m}\right)+m
$$

As $x$ increases, (7.2) says that the next zero is of $u_{2}$ and then since $W$ has no zeros, zeros of $u_{1}$ and $u_{2}$ must alternate. So for $c>x_{m}, N(c) \equiv N_{u_{2}}(c)-N_{u_{1}}(c)$ alternates between $m$ and $m+1$ and since $\tau$ is assumed to be oscillatory at $E_{2}$ near $b$, we immediately get $\frac{\lim }{c \uparrow b} N(c)=m$.

If $W_{0}\left(u_{1}, u_{2}\right)=\infty$, let $x_{m}$ be the $m$ th zero. Then (7.1) and (7.2) still hold so $N\left(x_{m}\right)=m$. Since $u_{2}$ has zeros between any pair of zeros of $u_{1}, N(x) \geq m$ for any $x \geq x_{m}$, so $\frac{\lim }{c \uparrow b} N(x)=\infty$, as required.

If $\tau$ is non-oscillatory at $E_{2}$ near $b$, we first assume that $E_{1,2}$ are not eigenvalues. We need to show that the hare ends up further along than the tortoise. Without loss of generality we assume $u_{1,2}(x)>0$ for $x$ near $b$ and claim in addition that $u_{1} u_{2}$ is not $L_{1}$ near $b$. If $u_{1}<u_{2}$ or $u_{2}<u_{1}$ eventually near $b$, we are done since $u_{1} u_{2}>u_{1}^{2}$ or $u_{1} u_{2}>u_{2}^{2}$ for $x$ near $b$ and $u_{j} \notin L^{2}$ near $b$. In fact, by hypothesis, $u_{j} \in L^{2}$ near $a$ and since $E_{j}$ are not eigenvalues and $\tau$ is limit point at $b, u_{j}$ cannot be $L^{2}$ near $b$. Otherwise we can find two points $x_{0}$ and $x_{1}$ close to $b$ such that $W\left(u_{1}, u_{2}\right)\left(x_{0}\right) \geq 0$ and $W\left(u_{1}, u_{2}\right)\left(x_{1}\right) \leq 0$, contradicting (2.1). But $u_{1} u_{2}$ not $L_{1}$ near $b$ together with (2.1) implies that $u_{2}^{\prime} / u_{2}>u_{1}^{\prime} / u_{1}$ for $x$ near $b$ which, by monotonicity of $\cot (\cdot)$, yields that the hare ends up ahead. 
It remains to treat the case where $E_{1,2}$ could be eigenvalues. Choose $E^{\prime}<E^{\prime \prime}$ with $u\left(E^{\prime}\right)$ (resp. $u\left(E^{\prime \prime}\right)$ ) equal to $\psi_{-}\left(E^{\prime}\right)$ (resp. $\left.\psi_{-}\left(E^{\prime \prime}\right)\right)$ the corresponding wave functions. Next, choosing $E^{\prime}$ below the spectrum of $H$ (implying that $u\left(E^{\prime}\right)$ has no zeros by Corollary 2.4) shows that the number of zeros of $u\left(E^{\prime \prime}\right)$ equals the number of eigenvalues below $E^{\prime \prime}$ (compare Corollary 2.4), that is, equals $\operatorname{dim} \operatorname{Ran} P_{\left(-\infty, E^{\prime \prime}\right)}(H)$ if $E^{\prime \prime}$ is not an eigenvalue. Theorem 2.1 then covers the case where $E^{\prime \prime}$ is an eigenvalue. Applying this to $E^{\prime \prime}=E_{1}$ and $E^{\prime \prime}=E_{2}$ proves (1.5b) since

$$
\operatorname{dim} \operatorname{Ran} P_{\left(-\infty, E^{\prime \prime}\right)}(H)-\operatorname{dim} \operatorname{Ran} P_{\left(-\infty, E^{\prime}\right)}(H)=\operatorname{dim} \operatorname{Ran} P_{\left[E^{\prime}, E^{\prime \prime}\right)}(H) .
$$

Finally, we want to consider the relation to the density of states. Given an SL operator $H$, let $H_{(L)}^{D}$ be the operator on $[-L, L]$ with Dirichlet boundary conditions. If the limit exists, we define the integrated density of states (ids), $k(E)$, by the limit:

$$
k(E)=\lim _{L \rightarrow \infty}(2 L)^{-1} \operatorname{dim} \operatorname{Ran} P_{(-\infty, E)}\left(H_{(L)}^{D}\right) .
$$

Theorem 7.6. Suppose $H$ is such that the ids exists for all E. Let $E_{1}<E_{2}$ and suppose $\tau u=E_{1} u, \tau \mathrm{V}=E_{2} \mathrm{~V}$. Let $W_{(L)}$ be the number of zeros of $W\left(u_{1}, u_{2}\right)$ in $[-L, L]$. Then

$$
\lim _{L \rightarrow \infty}(2 L)^{-1} W_{(L)}=k\left(E_{2}\right)-k\left(E_{1}\right)
$$

Proof. By Theorem 7.2 and Theorem 1.3, $\left|W_{(L)}-\operatorname{dim} \operatorname{Ran} P_{\left(E_{1}, E_{2}\right)}\left(H_{(L)}^{D}\right)\right| \leq 2$, so the result follows from

$$
\lim _{L \rightarrow \infty}(2 L)^{-1} \operatorname{dim} \operatorname{Ran} P_{\left(E_{1}, E_{2}\right)}\left(H_{(L)}^{D}\right)=k\left(E_{2}\right)-k\left(E_{1}\right) .
$$

Acknowledgments. F. G. is indebted to the Department of Mathematics at Caltech for its hospitality and support during the summer of 1994 and in February and March of 1995, where some of this work was done.

Appendix: Associated quadratic forms The purpose of this appendix is to clarify some form domain questions which arise due to our general conditions on the local behavior on $r, p$, and $q$ in (1.1).

We'll consider regular SL operators and hence assume $(a, b) \subset \mathbb{R}$ to be a finite interval with

$$
r, p^{-1}, q \in L^{1}((a, b)) \quad \text { real-valued and } r, p>0 \text { a.e. on }(a, b)
$$


Next, define in $L^{2}((a, b) ; r d x)$ the following linear operators

$$
\begin{gathered}
\left(H_{\alpha, \beta}^{0} u\right)(x)=-r(x)^{-1}\left(p(x) u^{\prime}(x)\right)^{\prime}, \\
D\left(H_{\alpha, \beta}^{0}\right)=\left\{u \in L^{2}((a, b) ; r d x) \mid u, p u^{\prime} \in A C([a, b]), r^{-1}\left(p u^{\prime}\right)^{\prime} \in L^{2}((a, b) ; r d x),\right. \\
\left.\left(p u^{\prime}\right)(a)+\alpha u(a)=\left(p u^{\prime}\right)(b)+\beta u(b)=0\right\}, \\
\alpha, \beta \in \mathbb{R} \cup\{\infty\}
\end{gathered}
$$

(here $\alpha=\infty$ denotes a Dirichlet boundary condition $u(a)=0$ and similarly at $b$ ),

$$
\begin{gathered}
S_{\alpha, \beta} u=s u, \quad(s u)(x)=(p(x) / r(x))^{1 / 2} u^{\prime}(x), \quad \alpha, \beta \in\{0, \infty\}, \\
D\left(S_{\alpha, \beta}\right)=\left\{u \in L^{2}((a, b) ; r d x) \mid u \in A C([a, b]), s u \in L^{2}((a, b) ; r d x),\right. \\
u(a)=0 \text { if } \alpha=\infty, u(b)=0 \text { if } \beta=\infty\}, \\
S_{\alpha, \beta}^{+} u=s^{+} u, \quad\left(s^{+} u\right)(x)=-r(x)^{-1}\left[(p(x) r(x))^{1 / 2} u(x)\right]^{\prime}, \quad \alpha, \beta \in\{0, \infty\}, \\
D\left(S_{\alpha, \beta}^{+}\right)=\left\{u \in L^{2}((a, b) ; r d x) \mid(p r)^{1 / 2} u \in A C([a, b]), s^{+} u \in L^{2}((a, b) ; r d x),\right. \\
\left((p r)^{1 / 2} u\right)(a)=0 \text { if } \alpha=0, \\
\left.\left((p r)^{1 / 2} u\right)(b)=0 \text { if } \beta=0\right\},
\end{gathered}
$$

and the form

$$
R_{\alpha, \beta}^{0}(u, \mathrm{v})=\left\langle S_{\alpha, \beta} u, S_{\alpha, \beta} \mathrm{V}\right\rangle, \quad \mathcal{D}\left(R_{\alpha, \beta}^{0}\right)=D\left(S_{\alpha, \beta}\right), \quad \alpha, \beta \in\{0, \infty\}
$$

$\left(\langle\cdot, \cdot\rangle\right.$ the scalar product in $\left.L^{2}((a, b) ; r d x)\right)$.

LEMmA A.1. (i) $S_{\alpha, \beta}=\left(S_{\alpha, \beta}^{+}\right)^{*}$ and $S_{\alpha, \beta}^{+}=S_{\alpha, \beta}^{*}$ for all $\alpha, \beta \in\{0, \infty\}$.

(ii) $H_{\alpha, \beta}^{0}=S_{\alpha, \beta}^{*} S_{\alpha, \beta}, \alpha, \beta \in\{0, \infty\}$.

Proof. Define

$$
\begin{aligned}
K: L^{2}((a, b) ; r d x) \rightarrow D\left(S_{\infty, 0}\right) & & K^{+}: L^{2}((a, b) ; r d x) \rightarrow D\left(S_{0, \infty}^{+}\right) \\
g \mapsto \int_{a}^{x} \frac{g(y) r(y) d y}{(p(y) r(y))^{1 / 2}} & & g \mapsto(p r)(x)^{-1 / 2} \int_{a}^{x} g(y) r(y) d y
\end{aligned} .
$$

A straightforward calculation verifies $(K g)(a)=0, s K g=g$ and $\left((p r)^{1 / 2} K^{+} g\right)(a)=$ $0, s^{+} K^{+} g=g$.

We only show $S_{\alpha, \beta}^{*}=S_{\alpha, \beta}^{+}$, the case $\left(S_{\alpha, \beta}^{+}\right)^{*}=S_{\alpha, \beta}$ being analogous. Moreover, since $S_{\infty, \infty} \subseteq S_{\alpha, \beta}$ implies $S_{\alpha, \beta}^{*} \subseteq S_{\infty, \infty}^{*}$ we only concentrate on proving $S_{\infty, \infty}^{*}=$ $S_{\infty, \infty}^{+}$, the rest following from an additional integration by parts.

An integration by parts proves $S_{\infty, \infty}^{+} \subseteq S_{\infty, \infty}^{*}$. Conversely, let $f \in D\left(S_{\infty, \infty}^{*}\right)$ and set $g=K^{+} S_{\infty, \infty}^{*} f$. Then

$$
\int_{a}^{b}(\bar{f}-\bar{g})\left(S_{\infty, \infty} h\right) r d x=\int_{a}^{b}\left(S_{\infty, \infty}^{*} \bar{f}-s^{+} \bar{g}\right) h r d x=0
$$


for all $h \in D\left(S_{\infty, \infty}\right)$. Thus, $\operatorname{Ran}\left(S_{\infty, \infty}\right)$ is a subset of the kernel of the linear functional $k \mapsto\langle f-g, k\rangle$. But $\operatorname{Ran}\left(S_{\infty, \infty}\right)=\left\{(p r)^{-1 / 2}\right\}^{\perp}$ (since $g \in \operatorname{Ran}\left(S_{\infty, \infty}\right)$ is equivalent to $(K g)(b)=0)$ and hence $f=g+c(p r)^{-1 / 2} \in D\left(S_{\infty, \infty}^{+}\right)$for some constant $c$ proving $S_{\infty, \infty}^{*} \subseteq S_{\infty, \infty}^{+}$and hence (i).

By inspection, we obtain $D\left(S_{\alpha, \beta}^{+} S_{\alpha, \beta}\right)=\left\{u \in D\left(S_{\alpha, \beta}\right) \mid S_{\alpha, \beta} u \in D\left(S_{\alpha, \beta}^{+}\right)\right\}=$ $D\left(H_{\alpha, \beta}^{0}\right)$ since $p u^{\prime} \in A C([a, b])$ implies $(p / r)^{1 / 2} u^{\prime}=(p r)^{-1 / 2}\left(p u^{\prime}\right) \in L^{2}((a, b) ; r d x)$ and $S_{\alpha, \beta}^{+} S_{\alpha, \beta} u=H_{\alpha, \beta}^{0} u$. This fact together with (i) proves (ii).

Furthermore, we introduce the forms

$$
\begin{gathered}
Q_{q / r}(u, \mathrm{v})=\int_{a}^{b} q(x) r(x)^{-1} \overline{u(x)} \mathrm{v}(x) r(x) d x, \\
\mathcal{D}\left(Q_{q / r}\right)=\left\{u \in L^{2}((a, b) ; r d x) \mid(q / r)^{1 / 2} u \in L^{2}((a, b) ; r d x)\right\},
\end{gathered}
$$

and

$$
\begin{gathered}
q_{\alpha, \beta}(u, \mathrm{v})=\tilde{\beta} \overline{u(b)} \mathrm{v}(b)-\tilde{\alpha} \overline{u(a)} \mathrm{v}(a), \quad \mathcal{D}\left(q_{\alpha, \beta}\right)=A C([a, b]), \\
\tilde{\alpha}=\left\{\begin{array}{l}
\alpha, \quad \alpha \in \mathbb{R} \\
0, \quad \alpha=\infty
\end{array}, \quad \tilde{\beta}=\left\{\begin{array}{c}
\beta, \quad \beta \in \mathbb{R} \\
0, \quad \beta=\infty
\end{array}, \quad \alpha, \beta \in \mathbb{R} \cup\{\infty\} .\right.\right.
\end{gathered}
$$

Finally, we set

$$
\begin{gathered}
Q_{\alpha, \beta}^{0}=R_{\widehat{\alpha}, \widehat{\beta}}^{0}+q_{\alpha, \beta}, \quad \mathcal{D}\left(Q_{\alpha, \beta}^{0}\right)=D\left(S_{\widehat{\alpha}, \widehat{\beta}}\right), \\
\widehat{\alpha}=\left\{\begin{array}{ll}
0, & \alpha \in \mathbb{R} \\
\infty, & \alpha=\infty
\end{array}, \quad \widehat{\beta}=\left\{\begin{array}{ll}
0, & \beta \in \mathbb{R} \\
\infty, & \beta=\infty
\end{array}, \quad \alpha, \beta \in \mathbb{R} \cup\{\infty\} .\right.\right.
\end{gathered}
$$

and

$$
Q_{\alpha, \beta}=Q_{\alpha, \beta}^{0}+Q_{q / r}, \quad \mathcal{D}\left(Q_{\alpha, \beta}\right)=D\left(S_{\widehat{\alpha}, \widehat{\beta}}\right), \quad \alpha, \beta \in \mathbb{R} \cup\{\infty\}
$$

Lemma A.2. (i) $q_{\alpha, \beta}$ is infinitesimally form bounded with respect to $Q_{0,0}^{0}$. (ii) $Q_{q / r}$ is relatively form compact with respect to $Q_{\alpha, \beta}^{0}, \alpha, \beta \in \mathbb{R} \cup\{\infty\}$.

Proof. (i) Since for arbitrary $c \in[a, b]$ and $u \in D\left(S_{0,0}\right)$,

$$
|u(c)|^{2}=\left|u(x)^{2}-2 \int_{c}^{x} u(t) u^{\prime}(t) d t\right| \leq|u(x)|^{2}+\int_{a}^{b}\left|u(t) u^{\prime}(t)\right| d t,
$$


one infers (after integrating from $a$ to $b$ ) for any $\epsilon>0$,

$$
\begin{aligned}
\|u\|_{\infty}^{2} & \leq(b-a)^{-1}\|u\|_{2}^{2}+2 \int_{a}^{b} \frac{|u(t)|}{(\epsilon p(t) / 2)^{1 / 2}}(\epsilon p(t) / 2)^{1 / 2}\left|u^{\prime}(t)\right| d t \\
& \leq(b-a)^{-1}\|u\|_{2}^{2}+\int_{a}^{b}\left(\frac{2}{\epsilon} \frac{|u(t)|^{2}}{p(t)}+\frac{\epsilon}{2} p(t)\left|u^{\prime}(t)\right|^{2}\right) d t .
\end{aligned}
$$

Since $0<p^{-1} \in L^{1}((a, b))$, we can find a $\delta(\epsilon)$ such that $\int_{I_{\delta(\epsilon)}} p(t)^{-1} d t \leq \frac{\epsilon}{4}$ with $I_{\delta(\epsilon)}=\{x \in(a, b) \mid p(x) \leq \delta(\epsilon)\}$. Thus,

$$
\int_{a}^{b} \frac{|u(t)|^{2}}{p(t)} d t=\int_{I_{\delta(\epsilon)}} \frac{|u(t)|^{2}}{p(t)} d t+\int_{(a, b) \backslash I_{\delta(\epsilon)}} \frac{|u(t)|^{2}}{p(t)} d t \leq \frac{\epsilon}{4}\|u\|_{\infty}^{2}+\frac{1}{\delta(\epsilon)}\|u\|_{2}^{2}
$$

and one obtains from (A.3),

$$
\|u\|_{\infty}^{2} \leq 2\left[(b-a)^{-1}+2(\epsilon \delta(\epsilon))^{-1}\right]\|u\|_{2}^{2}+\epsilon Q_{0,0}^{0}(u, u)
$$

completing the proof of (i).

(ii) Let $G_{\alpha, \beta}^{0}(z, x, y)$ denote the Green's function of $H_{\alpha, \beta}^{0}, \alpha, \beta \in \mathbb{R} \cup\{\infty\}$, that is,

$$
\left(\left(H_{\alpha, \beta}^{0}-z\right)^{-1} u\right)(x)=\int_{a}^{b} G_{\alpha, \beta}^{0}(z, x, y) u(y) r(y) d y, \quad z \in \mathbb{C} \backslash \mathbb{R} .
$$

Then $|q / r|^{1 / 2}\left(H_{\alpha, \beta}^{0}-z\right)^{-1}|q / r|^{1 / 2} \in \mathfrak{B}_{2}\left(L^{2}((a, b) ; r d x)\right)\left(\mathfrak{B}_{2}(\cdot)\right.$ the set of HilbertSchmidt operators) since

$$
\begin{aligned}
\left\||q / r|^{1 / 2}\left(H_{\alpha, \beta}^{0}-z\right)^{-1}|q / r|^{1 / 2}\right\|_{2}^{2} & =\int_{a}^{b} \int_{a}^{b} \frac{|q(x)|}{r(x)}\left|G_{\alpha, \beta}^{0}(z, x, y)\right|^{2} \frac{|q(y)|}{r(y)} r(x) r(y) d x d y \\
& \leq M(z)\left[\int_{a}^{b}|q(x)| d x\right]^{2}
\end{aligned}
$$

using the fact that $\left|G_{\alpha, \beta}^{0}(z, \cdot, \cdot)\right|$ is bounded on $(a, b) \times(a, b)$.

Thus the forms $Q_{\alpha, \beta}$ in (A.2) are densely defined, closed, and bounded from below ([12], section VI.1). We denote by $H_{\alpha, \beta}$ the uniquely associated selfadjoint operators bounded from below guaranteed by the KLMN theorem ([12], 
theorem VI.2.1; [16], theorem X.17). The following theorem identifies $H_{\alpha, \beta}$ as the usual regular SL operators (with separated boundary conditions).

THEOREM A.3. $H_{\alpha, \beta}$ associated with $Q_{\alpha, \beta}$ is given by

$$
\begin{aligned}
& \left(H_{\alpha, \beta} u\right)(x)=r(x)^{-1}\left[-\left(p(x) u^{\prime}(x)\right)^{\prime}+q(x) u(x)\right], \\
& D\left(H_{\alpha, \beta}\right)=\left\{u \in L^{2}((a, b) ; r d x) \mid u, p u^{\prime} \in A C([a, b]),\right. \\
& r^{-1}\left(-\left(p u^{\prime}\right)^{\prime}+q u\right) \in L^{2}((a, b) ; r d x), \\
& \left.\left(p u^{\prime}\right)(a)+\alpha u(a)=\left(p u^{\prime}\right)(b)+\beta u(b)=0\right\}, \\
& \alpha, \beta \in \mathbb{R} \cup\{\infty\} \text {. }
\end{aligned}
$$

Proof. It suffices to consider the Dirichlet case $\alpha=\beta=\infty$, the other cases being similar. Denote by $\widehat{H}_{\infty, \infty}$ the operator defined in (A.5) for $\alpha=\beta=\infty$ and by $H_{\infty, \infty}$ the unique operator associated with $Q_{\infty, \infty}$. Choose $u \in \mathcal{D}\left(Q_{\infty, \infty}\right)$ and $\mathrm{v} \in D\left(\widehat{H}_{\infty, \infty}\right)$. Then an integration by parts yields

$$
Q_{\infty, \infty}(u, \mathrm{v})=\left\langle u, \widehat{H}_{\infty, \infty} \mathrm{V}\right\rangle
$$

Thus $\widehat{H}_{\infty, \infty} \subseteq H_{\infty, \infty}$ by Corollary VI.2.4 of [12] and hence $\widehat{H}_{\infty, \infty}=H_{\infty, \infty}$ since $\widehat{H}_{\infty, \infty}$ is self-adjoint.

Remark. It follows from the above theorem that for arbitrary SL operators $H$ (not necessarily regular), elements $u \in L^{2}((a, b) ; r d x)$ which satisfy

$$
u \in A C_{\mathrm{loc}}((a, b)), \quad(p / r)^{1 / 2} u^{\prime} \in L_{\mathrm{loc}}^{2}((a, b) ; r d x)
$$

and which are in the domain of $H$ near $a$ and $b$, are in the form domain of $H$. Moreover, let $u(x), \mathrm{v}(x)$ be as in (A.6) and in $D(H)$ for $x \leq c$ and $x \geq d$, then

$$
\begin{gathered}
Q_{H}(u, \mathrm{v})=\int_{(a, b) \backslash(c, d)} \overline{u(x)}(\tau \mathrm{v})(x) d x+\overline{u(d)}\left(p \mathrm{v}^{\prime}\right)(d)-\overline{u(c)}\left(p \mathrm{v}^{\prime}\right)(c) \\
\left.\quad+\int_{c}^{d}\left[\overline{u^{\prime}(x)}\left(p \mathrm{v}^{\prime}\right)(x)+q(x) \overline{u(x)} \mathrm{V}(x)\right)\right] d x .
\end{gathered}
$$

In fact, take $u$ as in (A.6) and in $D(H)$ for $x \leq c$ and $x \geq d$. Consider the operator $\tilde{H}_{\alpha, \beta}$ associated with $\tau$ and boundary conditions induced by $u$ on the finite interval $(c, d)$ (cf. (5.1)). Since $u \uparrow_{(c, d)} \in \mathcal{D}\left(\tilde{Q}_{\alpha, \beta}\right)\left(\tilde{Q}_{\alpha, \beta}\right.$ the form of $\left.\tilde{H}_{\alpha, \beta}\right)$, we can pick a sequence $\tilde{u}_{n}$ in $D\left(\tilde{H}_{\alpha, \beta}\right)$ such that $\left\|\tilde{u}_{n}-u \uparrow_{(c, d)}\right\|_{2} \rightarrow$ 0 and $\left\langle\left(\tilde{u}_{n}-\tilde{u}_{m}\right), \tilde{H}_{\alpha, \beta}\left(\tilde{u}_{n}-\tilde{u}_{m}\right)\right\rangle \rightarrow 0$ (implying $\left\|\tilde{u}_{n}-u \uparrow_{(c, d)}\right\|_{\infty} \rightarrow 0$ by (A.4) and Lemma A.2). Extend $\tilde{u}_{n}$ to a function $u_{n}$ on $(a, b)$ by patching it with $u$ such that $u_{n} \in D(H)$ (which is possible since $u$ and $\tilde{u}_{n}$ satisfy the same boundary conditions at $c$ and $d$ ). By construction, $u_{n}$ satisfies $\left\|u_{n}-u\right\|_{2} \rightarrow 0$ and 
$\left\langle\left(u_{n}-u_{m}\right), H\left(u_{n}-u_{m}\right)\right\rangle \rightarrow 0$ and hence is in the form domain of $H$. This proves (A.6) and an integration by parts then proves (A.7).

Department of Mathematics, University of Missouri, Columbia, MO 65211

Electronic mail: MATHFG@MIZZOU1.MISSOURI.EDU

Division of Physics, Mathematics, and Astronomy, California Institute of TeChNOLOGy, Pasadena, CA 91125

DePartment of MATHEMATICS, University of Missouri, Columbia, MO 65211

Electronic mail: TESCHL@IRAM.RWTH-AACHEN.DE

Current address: Institut für Reine und Angewandte Mathematik, TH-Aachen, D-52056 Aachen, Germany

REFERENCES

[1] F. V. Atkinson, Discrete and Continuous Boundary Problems, Academic Press, New York, 1964.

[2] M. Bôcher, Leçons de Méthodes de Sturm, Gauthier-Villars, Paris, 1917.

[3] M. Buys and A. Finkel, The inverse periodic problem for Hill's equation with a finite-gap potential, J. Differential Equations 55 (1984), 257-275.

[4] W. A. Coppel Disconjugacy, Lecture Notes in Math., vol. 220, Springer-Verlag, New York, 1971.

[5] R. Courant and D. Hilbert, Methods of Mathematical Physics Volume I, Wiley, New York, 1989.

[6] N. Dunford and J. T. Schwartz, Linear Operators Part II: Spectral Theory, Wiley, New York, 1988.

[7] A. Finkel, E. Isaacson, and E. Trubowitz, An explicit solution of the inverse periodic problem for Hill's equation, SIAM J. Math. Anal. 18 (1987), 46-53.

[8] F. Gesztesy, B. Simon, and G. Teschl, Spectral deformations of one-dimensional Schrödinger operators, in preparation.

[9] P. Hartman, Differential equations with non-oscillatory eigenfunctions, Duke Math. J. 15 (1948), 697-709.

[10] A characterization of the spectra of one-dimensional wave equations, Amer. J. Math. 71 (1949), 915-920.

[11] P. Hartman and C. R. Putnam, The least cluster point of the spectrum of boundary value problems, Amer. J. Math. 70 (1948), 849-855.

[12] T. Kato, Perturbation Theory for Linear Operators, 2nd ed., Springer-Verlag, New York, 1980.

[13] K. Kreith, Oscillation Theory, Lecture Notes in Math., vol. 324, Springer-Verlag, New York, 1973.

[14] W. Leighton, On self-adjoint differential equations of second order, J. London Math. Soc. 27 (1952), $37-47$.

[15] M. A. Naimark, Linear Differential Operators Part II, Ungar, New York, 1968.

[16] M. Reed and B. Simon, Methods of Mathematical Physics II: Fourier Analysis, Self-Adjointness, Academic Press, New York, 1975.

[17] $\longrightarrow$ Methods of Mathematical Physics I: Functional Analysis, revised and enlarged ed., Academic Press, New York, 1980.

[18] W. T. Reid, Sturmian Theory for Ordinary Differential Equations, Springer-Verlag, New York, 1980.

[19] C. A. Swanson, Comparison and Oscillation Theory of Linear Differential Equations, Academic Press, New York, 1968.

[20] J. C. F. Sturm, Mémoire sur les équations différentielles linéaires du second ordre, J. Math. Pures Appl. 1 (1836), 106-186.

[21] J. Weidmann Linear Operators in Hilbert Spaces, Springer-Verlag, New York, 1980.

[22] - Spectral Theory of Ordinary Differential Operators, Lecture Notes in Math., vol. 1258, Springer-Verlag, New York, 1987. 